\title{
Hypopharyngeal carcinoma: a clinical study
}

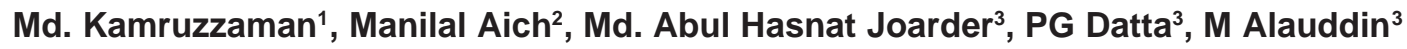

\begin{abstract}
A prospective study was carried out from 2001 to 2002 at Department of Otolaryngology and Head- Neck Surgery, BSMMU, DMCH, SSMC \& Mitford Hospital, Dhaka. Data were collected from 45 patients with histologically diagnosed hypopharyngeal carcinoma on the following variables 1) sociodemographics, 2) clinical manifestations, 3) sub-sites of carcinoma, 4) clinical staging, 5) time of diagnosis, 6) associated risk factors, and 8) patients general performance status at presentation was determined by the Karnofsky scoring system.

Results of this study showed that hypopharyngeal carcinoma affects fourth (60\%) and fifth (26.7\%) decade of life. It was found that male was highly predominant to develop hypopharyngeal carcinoma and ratio was 6:1. People with lower educational level and lower income group were found to be affected more by hypopharyngeal carcinoma. In this study the mean time elapsed from the appearance of the first symptom to the diagnosis of hypopharyngeal carcinoma was 4.11 months. This study found that the key symptom of hypopharyngeal carcinoma was dysphagia, pain, hoarseness and neck mass. 80 percent patient presented with palpable lymph node and all of them were in level II. Pyriform fossa was found to be the commonest site (80\%) affected by carcinoma. In this series 75 percent patient presented in stage III and stage IV. There is strong relation between staging and diagnostic delay $(p<0.05)$.

In this study 86.7 percent patients had history of smoking. Smoking was found significantly associated with hypopharyngeal carcinoma $(p<0.05)$. Betel nut chewing and tobacco chewing was found to be significant factors to develop hypopharyngeal carcinoma $(p<0.05)$. This study showed that there was no patient with good oral health. Bad and average orodental hygiene was found in 44.4 and 55.6 percent respectively. Statistically bad oral hygiene was significantly related to hypopharyngeal carcinoma $(p<0.05)$. Performance status of the patients at presentation was good and 77.8 percent of patients with hypopharyngeal carcinoma at diagnosis were capable of self-care.
\end{abstract}

\section{Introduction}

Carcinoma arising in this region present in an advanced state and the cure lies in early and accurate diagnosis. Hypopharyngeal carcinomas are considered indolent, silent tumors. They can present with a myriad of symptoms and clinical complaints. The silent nature of these tumors unfortunately causes a high number of patients to present with advanced disease $^{1}$.

The capacity of the hypopharynx allows considerable tumor growth before the passage of food is hindered, so most of the patients already have advanced tumors

1. Lecturer, Department of Community Medicine, Khulna Medical College

2. OSD (DGHS) Department of Otolaryngology-Head \& Neck Surgery, BSMMU

3. Professor, Department of Otolaryngology and HeadNeck Surgery, BSMMU

Address of correspondence: Md. Kamruzzaman, Lecturer, Department of Community Medicine Khulna Medical College, Khulna at presentation. Important neighboring structures, such as the larynx and prevertebral muscles are readily invaded, and the rich lymphatic drainage allows early metastatic spread to jugular, retropharyngeal, Para tracheal and mediastinal nodes.

Epidemiological study cannot satisfactorily define the risk factors to develop hypopharyngeal carcinoma, because of low incidence and different risk factors in different subtypes of hypopharyngeal carcinoma. Such as post-cricoid carcinoma are common in female.

Hypopharyngeal carcinoma (HPC) most often affects men in their fifth and sixth decade of life, with a consistently reported peak incidence at the age of $50-60$ years. The male to female ratio is overwhelmingly unfavorable for males ${ }^{2}$. But postcricoid carcinoma is common in women than men, and with wide geographical variation in its frequency ${ }^{3}$. HPC has been strongly associated with tobacco smoking and alcohol abuse 4 . Other risk factors include low consumption of fruits and vegetables ${ }^{5}$, poor oral hygiene ${ }^{6}$, deficient dental status ${ }^{7}$ and exposure to a 
wide variety of occupational carcinogenic agents ${ }^{8}$. Reports from Northern Europe used to indicate a link of Plummer-Vinson syndrome and other deficiency conditions (e.g. iron deficiency anemia, vitamin-A deficiency) to HPC in women?.

Smoking with drinking was multiplicatively greater than those for smoking or drinking in combined cases of hypopharyngeal carcinoma and esophageal carcinoma ${ }^{10}$. Smoking tobacco and chewing of betel nuts and tobacco leaves have got relation in the causation of pharyngeal malignancy. Lower standard of living, low income, overcrowding, poor orodental hygiene are risk factors in the development of pharyngeal malignancy, possibly by lowering the level of body immunity or due to chronic irritation. Spicy and hot foods contribute to the development of pharyngeal malignancy ${ }^{11}$.

Poor oral hygiene is believed to play a role as a risk factor for head neck cancer, especially for oral cancer. Maier $\mathrm{H}$ et al, 1993 performed a case control study involving 100 patients with squamous cell carcinoma of the upper aerodigestive tract and 214 age and sex matched control subjects with no known tumorous disease. In the tumour patients, bad oral hygiene and poor dental status proved to be significantly worse: the majority of the tumour patients seldom or never brushed their teeth and the frequency of dental visits was significantly lower. Tartar of $3 \mathrm{~mm}$ or more was found in $40.9 \%$ of the tumour patients and in $22 \%$ of the control subjects. In the tumour group, the incidence of decayed teeth was significantly higher compared with the control subjects. Chronic inflammation of the gingiva was observed in $28 \%$ of the tumour patients vs. $13.5 \%$ in the control group. Oral hygiene was negatively correlated with alcohol as well as with tobacco consumption. The social status of subjects also correlated with oral hygiene, which was found to be worst in subjects from the lower social strata ${ }^{7}$.

Genetic predisposition is an important risk factor for squamous cell carcinoma of the head and neck. First degree relatives of 105 patients with head neck cancer had 31 cases of cancer of the respiratory and upper digestive tract vs 10 cases in the control group (relative risk, $3.5 ; p=.0002)^{12}$. Multiple primary cancers of the head and neck are not rare. Aibara $\mathrm{R}$ et al have experienced 30 cases of multiple cancers from 1976 to 1989 . The incidence ranged from $3.6 \%$ to $8.9 \%$ with flexible criteria. The concept of "multicentric cancerization" was verified by the result that $60 \%$ of the additional cancers were head and neck, esophagus and lung. During follow-up studies of oropharyngeal, hypopharyngeal and laryngeal cancers, we have to examine the esophagus periodically due to high risk of occurrence of cancer ${ }^{13}$.

It is a general believe that premalignant condition is associated with carcinoma. Pathological changes such as acanthosis, basal cell hyperplasia, intra epithelial neoplasia, chronic oesophagitis koilocytosis and papillomatosis occur in the oral hypopharyngeal and other areas of the oesophageal squamous mucosa, in association with squamous carcinoma of the oesophagus ${ }^{14}$.

The key symptoms were pain, dysphagia, hoarseness, a painless neck mass, or a visible ulcerating lesion. Heamoptysis an unusual symptom, but can occur with tumours of the pyriform fossa and tumours of the posterior pharyngeal wall. Weight loss often occurs in the presence of significant disease ${ }^{15}$.

Hypopharyngeal carcinoma spread through submucosal invasion and local lymph node metastasis. Distant metastasis is usually rare. Submucosal invasion is much longer in the oral direction than in the anal direction. The longest invasion was observed by Murakami $Y$ et al in a case measured up to $22 \mathrm{~mm}$. the shortest invasion, on the contrarily was only $9 \mathrm{~mm}^{16}$. Robertson $\mathrm{MS}$ et al found 26 percent of hypopharyngeal carcinoma with a metastatic cervical node. Nodal metastases are related to cellular, nuclear and structural grading ${ }^{17}$. Clinical and statistical analysis proved that Carlon's grading, based on structural organization of the tumour, seen as a manifestation of its cellular cohesiveness, is a more discriminating system of malignancy than the others. The most organoid growth patterns do not present any node metastasis. they can be regarded as manifestation of "low grade malignancy tumour". The least organoid or non organoid growth patterns show higher levels of node metastasis. So structural grading could be the guideline to better management in terms of choice of surgical treatment ${ }^{18}$.

The chance survival decreases with increasing tumour size. much more however it will be affected by neck node metastasis. Ganzer $U$ et al experienced the survival rate for $\mathrm{pN} 1-3$ is less by half of pNo. therefore not only the TNM system but also the staging should be used for the classification of hypopharyngeal carcinoma. The main causes of death are lymph node relapse ${ }^{19}$. 
On average a 4-month period elapsed from the onset of symptoms to the histological diagnosis. The diagnostic delay was significantly shorter in patients with pain or visible organic changes (ulcerating lesions, neck masses) than in patients with functional symptoms (dysphagia and hoarseness) ${ }^{20}$.

Performance status is the only significant predictor of survival. Other host factors (age and sex) and all tumor factors ( site, T, N and M stage, Distant metastasis stage grouping, and histological grade of the tumour ) are not significant. Only performance status needs to be used as a stratification factor in trials of patients.

\section{Methodology}

It was a prospective study and collected data from 45 patients with histopathologically diagnosed hypopharyngeal carcinoma at Bangabandhu Sheikh Mujib Medical University, Dhaka Medical College Hospital and Sir Salimullah Medical College Hospital from 2001 to 2002 according to a prescribed data collection sheet. Data were collected on following variables 1) sociodemographics, 2) clinical manifestation, 3) sub-sites of carcinoma, 4) clinical staging, 5) time of diagnosis, 6) associated risk factors, and 8) patient's general performance status as determined by the Karnofsky scoring system. Data analysis was done by using SPSS version 10.0 and for significance pyriform fossa carcinoma was compared with other sub-sites hypopharyngeal carcinoma. Chi-square test was applied to find out the significance.

\section{Results}

There were 45 patients with histologically proved Hypopharyngeal carcinoma fulfilled the criteria and were taken in this study. Most of the Hypopharyngeal carcinoma presented between fourth and fifth decade. There is no subject below 38 years and over 60 years. Mean age was 47 years. Male persons were highly predominant to develop hypopharyngeal carcinoma and male-female ratio about 6:1.

There were no particular occupations, which are prone to develop Hypopharyngeal carcinoma but lower class physical worker or economically under privileged groups of the society were found to develop Hypopharyngeal carcinoma.

People with lower education were found to be affected by hypopharyngeal carcinoma. In this series 31 percent were illiterate and 47 percent completed their primary education. Lower income also associated with hypopharyngeal carcinoma. Three fourth of the subjects in this study earn less than five thousands taka monthly.

The mean time elapsed from the appearance of the first symptom to diagnosis of hypopharyngeal carcinoma was 4.11 months (range- 2-6 months). For Hypopharyngeal carcinomas 95.6 percent of patients exhibited dysphagia and mean duration of dysphagia was 16.16 weeks and range from 8 weeks to 25 weeks. Pain in form of referred otalgia or odynophagia was complained by 93.3 percent of patients. Mean duration of pain was 9.69 weeks and range from 4 weeks to 22 weeks.

Hoarseness was complained by 22.2 percent of patients. Mean duration of hoarseness was 6 weeks and ranging 4 weeks to 16 weeks. Neck mass is in form of direct extension or metastatic neck node were presented in 73.3 percent of patients. Duration of neck masses were ranging from 4 weeks to12 weeks in this study. Other clinical manifestation was weight loss (75.6 percent), anorexia (73.3 percent) and fatigue (75.6 percent).

Neck mass in form of direct extension or metastatic neck node was presented in 73.3 percent of patients. Duration of neck mass is ranging from 4 weeks to 12 weeks in this study. Few patients presented with haemoptysis and it is 13.3 percent of patients, duration of haemoptysis range from 4 weeks to 12 weeks.

73.3 percent subjects of this study gave history of anorexia. Mean duration of anorexia was 8 weeks and ranging from 6 weeks to 16 weeks. Fatigue was complained by 75.6 percent of subjects in this study group. Mean duration of fatigue was about 8 weeks and ranging from 4 weeks to 16 weeks.

Most common symptoms are dysphagia, pain and neck mass. Haemoptysis occur in few cases. Weight loss, anorexia and fatigue are equally in hypopharyngeal carcinoma. Pyriform fossa was the most common site in hypopharyngeal carcinoma and it was 80 percent. Post-cricoid and posterior wall carcinoma was comparatively rare. Their frequencies were 11 percent and 9 percent respectively.

Level II of lymph node group predilection site is for hypopharyngeal carcinoma. 80 percent patients present with palpable lymph node and all of them were in level II. In this study retropharyngeal and mediastinal nodes were not evaluated. $T_{1}$ and $T_{2}$ tumour were 80 
percent in this study. $T_{3}$ and $T_{4}$ were 13.3 and 6.7 percent respectively. Palpable lymph node size was upto $6 \mathrm{~cm}$. Only 8.9 percent patients had bilateral palpable lymph node in our series. There was no patient had distant metastasis at presentation of hypopharyngeal carcinoma in this study. Most of the cases present in advanced stage. Only 24.5 percent case presented in stage I and stage II in our series. 57.8 percent was in stage III and 17.8 percent was in stage IV. Histopathological study found that the growth in Hypopharynx was squamous cell carcinoma in 100 percent. Differentiations were well differentiated 11.1 percent, moderately differentiated 46.7 percent and poorly differentiated 42.2 percent. 6.7 percent patients were found to have a positive family history of headneck carcinoma. There was no single case had concomitant non head-neck cancer.

Smoking was found to have a significant relation with hypopharyngeal carcinoma. 86.7 percent had history of smoking. Their mean duration of smoking was 19.6 years and mean number of stick smoking was 11 per day. Alcohol consumption was found to have nonsignificant relation in three subjects. Only 15.6 percent subjects were alcoholic. In this series 48.89 percent subjects were found to have history of betel nut chewing. Rest 51.11 percent was devoid of betel nut chewing. History of tobacco chewing was found in 37.77 percent patients. 62.22 percent had no history of tobacco chewing. There was no good oral health. Bad and average orodental hygiene was found in 44.4 percent and 55.6 percent respectively. 80 percent of our patients had some extent of anaemia. There was no anaemia in 9 percent of the subjects in this series.

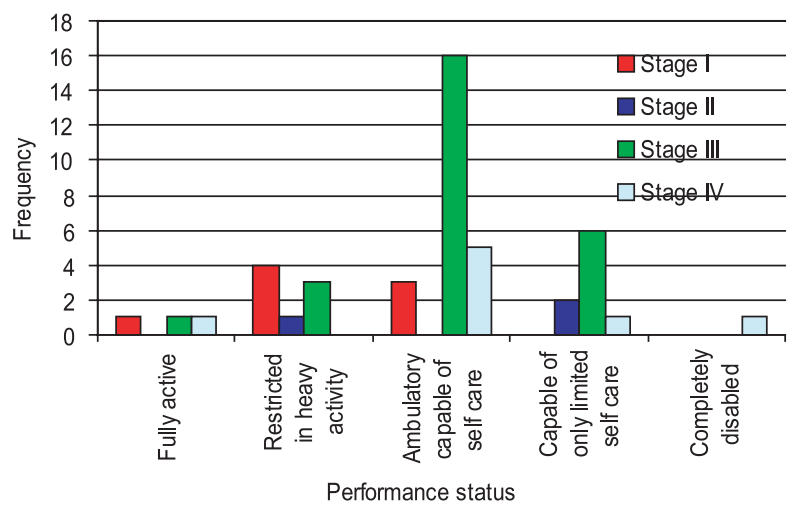

Fig.-1: Staging and performance status at presentation

Performance status at presentation was good in hypopharyngeal carcinoma. Though advanced stage carcinoma at presentation was 75.6 percent in this series karnofsky score was expected to be lower. But 77.8 percent patients with hypopharyngeal carcinoma at presentation were capable of self-care.

\section{Discussion}

In the present study the hypo pharyngeal carcinoma was most commonly seen in the age group of fifth and sixth decade of life. This is consistent with the finding of $A E$ Uzcudn et al, who found that hypopharyngeal carcinoma most often affects man in their fifth and sixth decade of life, with a consistently reported peak incidence at the age of 50-60 years ${ }^{2}$.

In this study the sex distribution revealed that male were highly predominant to develop hypopharyngeal carcinoma and the male to female ratio was about 6:1. AE Uzcudn et al also found similar ratio and opined that in hypopharyngeal carcinoma the male to female ratio is overwhelmingly unfavourable for men.

This study showed that the people with lower education were found to be affected more by hypopharyngeal carcinoma. The relation between education and hypopharyngeal carcinoma is statistically significant $(p<0.05)$. Lower income was also associated with hypopharyngeal carcinoma $(p<0.05)$. Three fourth of the subjects in this study earn less than Taka five thousand monthly. Fraije found that the lack of awareness, lower socioeconomic and poorly compliant population is susceptible to develop hypopharyngeal carcinoma ${ }^{21}$. Rahman $M Z$ in a Bangladeshi series explained that lower standard of living, low income, overcrowding, poor orodental hygiene are risk factors in the development of pharyngeal malignancy ${ }^{11}$.

Time lapse for diagnosis of hypopharyngeal carcinoma is an important factor for prognosis. In this study the mean time elapsed from the appearance of the first symptom to diagnosis of Hypopharyngeal carcinoma was 4.11 months. Wolfensberger et al also found that on average a 4-month period elapsed from the onset of symptoms to the histological diagnosis. The diagnostic delay was significantly shorter in patients with pain or visible organic changes (ulcerating lesions, neck mass) than in patients with functional symptoms (dysphagia and hoarseness) ${ }^{20}$.

The study showed that in hypopharyngeal carcinoma 95.6 percent patients present with dysphagia and mean duration of dysphagia was about 16 weeks. Pain was complained by 93.3 percent of patients and mean duration of pain was 9.69 weeks. Hoarseness was 
complained by 77.8 percent of patients. Neck mass in the form of direct extension or metastatic neck node was seen in 73.3 percent of patients. Haemoptysis, weight loss and fatigue were also complained by significant amount of patients. Wolfensberger $M$ et al (1994) opined that pain, dysphagia, hoarseness, a painless neck mass or visible ulcerating lesion is key point to diagnose Hypopharyngeal carcinoma. In this study pyriform fossa was the commonest site in hypopharyngeal carcinoma and it was 80 percent. Post-cricoid and posterior wall carcinoma was comparatively rare, frequency were 11 percent and 9 percent respectively ${ }^{20}$. JC Watkinson mentioned the most common tumour was that of the pyriform fossa, which forms between a half and two-thirds of the total ${ }^{15}$. Randall et al in their review found that postcricoid cancer formed up to $50 \%$ of hypopharyngeal cancer in the UK and Canada ${ }^{3}$.

In this study 80 percent patients presented with palpable lymph node and all of them were in level II . Robertson MS et al found that 26 percent of hypopharyngeal carcinoma presented with a metastatic cervical node. Steiner Wet al (1994) in a study found that $90 \%$ of the cases presented with neck metastases $(\mathrm{pN}+)$ and mostly in level II and III. In this study retropharyngeal and mediastinal lymph node were not evaluated ${ }^{17}$.

In this series T1 was 44.4 percent and $\mathrm{T} 2$ tumour was 35.6 percent. T3 and T4 were 13.3 and 6.7 percent respectively. Palpable lymph node size was up to 6 $\mathrm{cm}$. Only 8.9 percent patients had palpable bilateral lymph. There were no distant metastases in this series. Murakami Yet al found that hypopharyngeal carcinoma spread through submucosal invasion and local lymph node metastasis. Distant metastasis was usually rare ${ }^{16}$. Submucosal invasion is much longer in the oral direction than in the anal direction.

This study showed that most of the cases (75.6 percent) of hypopharyngeal carcinoma presented in advanced stage. There is significant relation between staging and diagnostic delay $(p<0.05)$. Steiner $W$ et al in a study found almost similar percent $(71.4 \%)$ of the patient presented in advanced stage ${ }^{22}$.

In the present study the histopathological type of hypopharyngeal carcinoma was squamous cell carcinoma in all cases. Differentiations were like well differentiated in 11.1 percent, moderately differentiated in 46.7 percent and poorly differentiated in $\mathbf{4 2 . 2}$ percent. Mazzone A mentioned that nodal metastases are related to cellular, nuclear and structural grading ${ }^{18}$. Clinical and statistical analysis proved that Carlon's grading based on structural organization of the tumour seen as a manifestation of its cellular cohesiveness is a more discriminating system of malignancy than others. The most organoid growth patterns do not present any node metastasis; they can be regarded as manifestation of "low grade malignancy tumour". The least organoid or non-organoid growth patterns show higher levels of lymph node metastasis. So grading could be the guideline to better management in terms of surgical treatment.

In this study 86.7 percent patients had history of smoking. Mean duration of smoking was 19.6 years and mean number of stick smoking was 11 per day. So smoking is significantly associated with hypopharyngeal carcinoma $(p<0.05)$. Rahman $M Z$ found smoking tobacco and chewing of Betel nuts and tobacco leaves have got relation in the causation of pharyngeal malignancy ${ }^{11}$. T. Takezaki showed that hypopharyngeal carcinoma was multiplicatively greater in person had a history of smoking with drinking than those for smoking or drinking ${ }^{10}$.

In this study only 15.6 percent subjects were alcoholic. Forty two percent subjects were found to have history of Betel nut chewing. Mean duration of Betel nut chewing was about 8 years. Relation of Betel nut chewing with hypopharyngeal carcinoma is significant $(p<0.05)$. History of tobacco chewing was found in 31 percent patients. Tobacco chewing is also a significant factor to develop hypopharyngeal carcinoma.

This study showed that there was no patient with good oral health. Bad and average orodental hygiene was found in 44.4 percent and 55.6 percent respectively. Bad oral hygiene significantly related with hypopharyngeal carcinoma $(p<0.05)$. Maeir $\mathrm{H}$ et al found that oral hygiene and dental status is proved be significantly worse in the tumour patients ${ }^{7}$. The majority of tumour patients seldom or never brushed their teeth and frequency of dental visits was significantly lower. Tartar of $3 \mathrm{~mm}$ or more was found in $40.9 \%$ of the tumour patients and in $22 \%$ of the control subjects.

In this series performance status of the patients at presentation was good and 77.8 percent of patients with hypopharyngeal carcinoma at diagnosis were capable of self-care, although 75.6 percent of hypopharyngeal carcinoma presented at advanced stage and 24.4 percent presented at early stage 
$(p<0.05)$. There is significant relation between staging and performance status at presentation of hypo pharyngeal carcinoma $(p<0.05)$. It is usual believe that survival in head-neck malignancy is depend on some host factors and some tumour factors like staging and histological type. Contrary to that Stell PM described performance status is the only significant predictor of survival. Other host factors (age and sex) and all tumour factors (site, TNM stage, distant metastasis, stage grouping, and histological grade of tumour\} are not significant. Performance status of hypopharyngeal carcinoma at presentation in this series is good ${ }^{23}$.

\section{Conclusion}

We can conclude that people with lower socioeconomic condition are mostly susceptible to develop hypopharyngeal carcinoma. Piriform fossa is the commonest site of affection in hypopharyngeal carcinoma. It can also be concluded that time elapsed between first clinical manifestations and conclusive diagnosis of hypopharyngeal carcinoma (4 months) is longer. Even then hypopharyngeal carcinoma presents with regional metastasis and level II lymph nodes are most commonly affected. Distant metastasis is rare in hypopharyngeal carcinoma. So earlier diagnosis is imperative in case of hypopharyngeal carcinoma.

We would also like to conclude that hypopharyngeal carcinoma should be suspected if a male patients aged between 40 to 60 years presented with dysphagia, neck node and had a history of smoking.

\section{References}

1. Mansfied EL, Cote DN. Hypopharyngeal carcinoma. J La State Med Soc, 1995; 147(11): 489-92.

2. Uzcudun AE, Fernandez PB, Sanchez JJ. Clinical feature of pharyngeal cancer: a retrospective study of 258 consecutive patients. The journal of laryngology and otology, 2001; Vol. 115: 112-118.

3. Randall P, Morton, Nicholus P, Melvor (1997). Tumours of the Hypopharynx. In: Scott-Brown's Otolaryngology. 6th edition. Vol 5. Laryngology and Head-neck Surgery. Edited by John Hibbert. London; Butterworths. PP-5/15/1-5/15/17.

4. Bloot WJ, McLaughlin, WinnDM, Austin DF, Greenberg RS, Preston-Martin S, Et al. Smoking and drinking in relation to oral and pharyngeal cancer. Cancer Res, 1998; 48: 3282-7.

5. Steinmetz K, PotterJD . Vegetables, fruitancer; (1) Epidemiology. Cancer causes Control, 1991; 2: 325-57.

6. Shapiro S, Castellana JV, Sprafka JM. Alcoholcontaining mouthwashes nad oropharyngeal cancer; a spurious association due to under ascertainement of confounders? Am J Epidemiol, 1996;144: 1091-5.

7. Maier H, Zoller J, Herrmann A, Kreiss M, Heller WD. Dental status and oral hygiene in patients with head and neck cancer. Otolaryngol head Neck Surg, 1993; 108(6): 655-61

8. Maier H, De Vries N, Snow GB (). Occupational factors in the aetilogy of head and neck cancer. Clin Otolaryngol, 1991; 16: 406-60.

9. Jensen OM. International variation in the incidence of cancer of the upper digestive and respiratory tract. Adv Otorhinolarygol, 1991; 46: 124-33.

10. Takezaki T, Shinoda M, Hatooka S, et all Subsitespecific risk factors for hypopharyngeal and esophageal cancer (Japan). Cancer causes and control, 2000; 11: 597-608.

11. Rahman MZ. Possible aetiological factors in pharyngeal malignancy- its relation to personal habits.Bangladesh J of Otolaryngology, 1999; 5(2): 28-31.

12. Copper MP, Jovanovic A, Nauta JJ, et all. Role of genetic factors in the etiology of squamous cell carcinoma of the head neck. Arch Otolaryngol Head Neck Surg, 1995; 121(2): 15760.

13. Aibara R, Yumoto E, Okamura $\mathrm{H}$, yanagihara $\mathrm{N}$. Multiple primary cancers of the head and neck. Nippon Jibiikoka Gakkai Kaiho 1990; 93(3): 381-7.

14. Ratnatunga NV, Edussuriya B. Mucosal changes in the upper gastrointestinal tract in Sri Lankan patients with squamous carcinoma of the oesophagus. Indian J Cancer, 1997;34(3):143-8

15. JC Watkinson, MN gaze, JA Wilson (2000). Tumours of the Hypopharynx. In: Head-Neck surgery. $4^{\text {th }}$ edition, Butterworth-Heinemann, Oxford. PP-335-356. 
16. Murakami $\mathrm{Y}$, Yanuda N, Murakami M dt all Correlation of proliferation index with submucosal invasion of hypopharyngeal cancer. Nippon Jibiinkoka Gakkai kaiho, 1990; 93(1): 71-9

17. Robertson MS, Snape L. The malignant gland in the neck as a presenting sign in head neck cancer. N Z Med J 1980; 22; 92(670):303-5

18. Mazzone A, Califano L, Agozzino L, et all. Anatomopathologic and clinical correlations of squamous carcinoma of the larynx and the hypopharynx. Acta Otorhinolaryngol Ital, 1990; 10(2): $111-9$

19. Ganzer U, Meyer-Breiting E, Ebbers J, Vsteen $\mathrm{KH}$. Effect of tumor size on lymph node metastasis and type of treatment on the prognosis of hypopharyngeal cancer. Laryngol Rhinol Otol (stuttg), 1982; 61(11): 622-8
20. Wolfensberger M, Schmid S, Schatzmann E. Clinical aspects in oral, pharyngeal and laryngeal carcinoma-results of an overall Swiss cohort study. Schweiz Med Wochenschr, 1994; 23; 124(16): 678-83

21. Freije J; Kumar JV. prevention of cancers of oral cavity and pharynx in new York state. N Y State dent J 2001; 67(3): 26-30

22. Steiner W, Stenglein C, Fietkau R, Sauerbrei W . Therapy of hypopharyngeal cancer. Part IV: Long-term results of transoral laser microsurgery of hypopharyngeal cancer. HNO, 1994; 42(3): 147-56

23. Stell PM. Survival time in end-stage head and neck cancer. Eur J Surg Oncol, 1989; 15(5): 40710. 